\title{
Analysis of emergency situations on the process of thermal power plants using mathematical apparatus of Petri nets
}

\author{
Sergey Trashchenkov, Vladimir Egorov \\ Pskov State University, Faculty of Electrical Engineering
}

\begin{abstract}
Article is dedicated to the issues of accident scenarios analysis on the process of thermal power plants. The theoretical foundations of the graphical and analytical representation of Petri nets are shown. The article describes the steam cycle process and listing of its technology protections. A state model for power unit equipment and technological protections under the influence of changes in critical process parameters (steam's temperature, pressure, level) is presented. Conclusions about the plans for further work in this direction are proposed.
\end{abstract}

Keywords: thermal power plants, risk analysis, Petri nets, technological protections.

\section{INTRODUCTION}

Thermal power plants are the basis of the generating industry in Russia and many other countries. Serious failures and accidents at thermal power plants not only lead to an aggravation of the electric power system operation, a possible deterioration in the quality and electricity shortage. Also power plant permanent equipment and ancillary equipment of thermal power plant can be damaged; building structures can be destroyed; staff can suffer. The process of electricity production at thermal power plants is a complex technology for energy conversion. This process is associated with a variety of potential hazards - burning fuel, using high-pressure steam; there are mechanisms with a large supply of mechanical energy, using grids and equipment from different voltage levels and others.

Over the past 40 years in the Soviet Union and the CIS 30 major accidents occurred with the failure of more than one power unit [1]. Over $90 \%$ accidence occurred due to failure of the main and auxiliary equipment. Such accidents do not bear the consequences of such accidents at nuclear power plants, but cause damage to millions of dollars.

Power plants are designed with a large reliability margin, enough to withstand a single failure of equipment or personnel, but we can see that serious accidents still occur. Causes of accidents on the thermal power plants are usually not single negative factor, but a combination of several of them. A careful study of all important, but low probability negative factors that can lead to damage and loss of human life is the basis of modern approaches to the safety management in the industry. Risk analysis is a component of safety management. Hazards are identified, risks and risk mitigation requirements are established, technical and organizational solutions for reducing the risk are found out during the procedure of risk analysis.

There are several quantitative and qualitative risk assessment methods that that are applied to designed and already operated facilities. It is usually difficult to quantify the risk of a complex system such as a process of power generation at the thermal power plant, consisting of hundreds of items. Even qualitative analysis is complicated by the structural complexity that cannot be reduced to the serial and parallel structures. Mathematical apparatus of modeling Petri nets is suitable for such systems. Petri nets are widely used in the simulation of dynamic discrete systems. Graphical programming language for controllers SFC (Sequential Function Chart) was created and is based on the formalism of Petri nets. Stochastic and fuzzy Petri net modifications allow to model systems with high levels of uncertainty.

The main system to counter the development of accidents is the emergency control schemes (technological protections). It is a complex control automatics, consisting of monitoring the critical parameters sensors, the logical unit controller and final elements that affect the process and prevent an accident.

This article is dedicated to the possibility of using Petri nets for modeling of emergencies on the process of thermal power plants. 


\section{MATERIALS AND METHODS}

A. Description of Petri net's formalism

Petri nets are bipartite directed multigraphs with two types of nodes (places and transitions). Petri nets are described by a mathematical expression

$C=\left(P, T, F, W, M_{0}\right)$

where $P=\left\{p_{1}, p_{2}, \ldots p_{n}\right\}$ is a set of places; $T=\left\{t_{1}, t_{2}\right.$ $\mathrm{t}_{\mathrm{m}}$ \} is a set of transitions; $\mathrm{F}$ is a set of arcs; $\mathrm{W}$ is an incidence-function; $\mathrm{M}_{0}$ is the initial marking. Arcs connect only nodes of different types

$\mathrm{F} \subset(\mathrm{P} \times \mathrm{T}) \cup(\mathrm{T} \times \mathrm{P})$

Arcs (and places) are input if ones connect places with transitions ( $\subseteq \mathrm{P} \times \mathrm{T}$ ) and are output if vice versa $(\mathrm{O} \subseteq \mathrm{T} \times \mathrm{P})$. One couple of nodes can be connected by more than one arc with different directions.

Graphically places are pictured by circles and transitions are pictured by rectangles. Places, transitions and arcs form the static structure of Petri nets.

Marking is responsible for the dynamic component of models based on Petri nets. It is a vector $\mathrm{M}=\left(\mathrm{m}_{1}\right.$, $\left.\mathrm{m}_{2}, \ldots \mathrm{m}_{\mathrm{k}}\right)$, where $\mathrm{m}_{\mathrm{i}}=\mathrm{M}\left(\mathrm{p}_{\mathrm{i}}\right)$. Marking changes discretely from the state $M_{0}$ to the state $M$ by the rules described below. Graphically marking are pictured by dots inside appropriate places. These dots are named "tokens". Each place can contain zero, one or few tokens.

The presence of tokens in places is a condition to make transition enabled. All places which are connected with arcs that lead to transition must have enough tokens to make it enabled. After that transition can be fired. Then transition is firing number of tokens equal number of input arcs disappears from appropriate places and tokens appear in places connected with firing transition equal number of output arcs. After transition had fired the Petri net got a new marking. Example of graphical presentation is shown on Fig.1.

There is one token in place p1. This token makes enabled at once two transitions (t1 and t2) but only one of them will fire. After firing transition $\mathrm{t} 1$ or $\mathrm{t} 2$ transition $t 4$ will be enabled because place $\mathrm{p} 4$ will have a token. Firing of transition $t 4$ will lead to situation when each of places p1, p2, p3 will get one token. Transitions t1, t2, t3 will be enabled. Transition $\mathrm{t} 3$ become enabled only because both of places $\mathrm{p} 2$ and p3 got tokens.

There is an analytic presentation of Petri net in addition to the graphical representation. In Fig. 1 Petri net has 4 transitions and 4 places. Transitions have mapping on sets of input and output places:

$$
I(t 1)=\{p 1\} \quad O(t 1)=\{p 4\}
$$

$$
I(t 2)=\{p 1\} \quad O(t 2)=\{p 4\}
$$

(1)

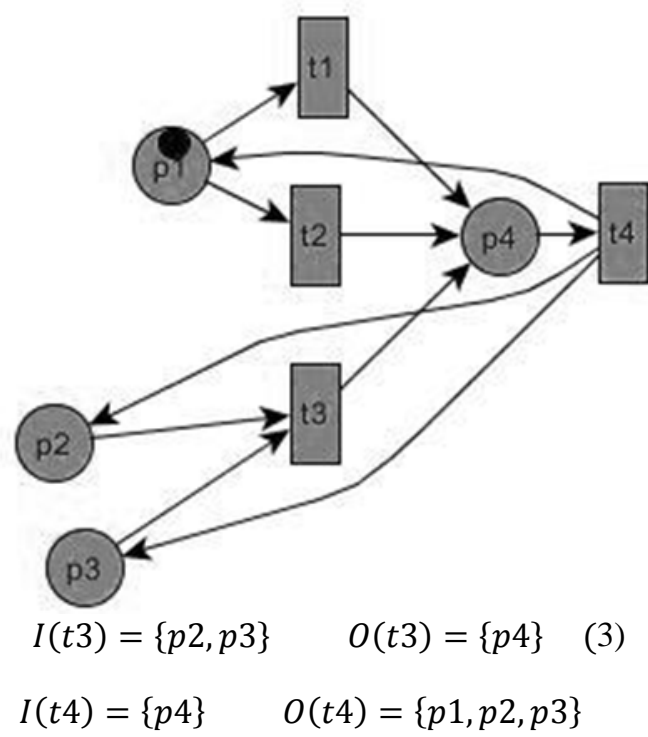

The incidence matrix can be contracted on the basis of mapping.

The incidence matrix has number of rows that equals the number of transitions and number of columns that equals the number of places. Every position in matrix places value $-1,0$ or 1 . If place $p_{i}$ is input to transition $\mathrm{t}_{\mathrm{j}}$ that position $[\mathrm{j}$, $\mathrm{i}]$ places value -1 . 1 is placed in the position in the case of place is output to transitions. 0 is placed in the position if there is no any connection between transition and place.

Incidence matrix for Petri net from Fig. 1 is shown in (4). Incidence matrix describes structure of Petri net. Dynamic changes are described by transition matrix (5) and marking matrix (6). Transition matrix shows transition which will fire next. Every position in transition matrix represents transition. In (5) t1 will fire and will change initial marking $\mathrm{M}_{0}$ (6) to marking $\mathrm{M}_{1}$.

Fig.1. Petri net example

$W=\left[\begin{array}{cccc}-1 & 0 & 0 & 1 \\ -1 & 0 & 0 & 1 \\ 0 & -1 & -1 & 1 \\ 1 & 1 & 1 & -1\end{array}\right]$

$\operatorname{Tr}_{1}=\left[\begin{array}{llll}1 & 0 & 0 & 0\end{array}\right]$

$M_{0}=\left[\begin{array}{llll}1 & 0 & 0 & 0\end{array}\right]$

Marking change and state change of Petri net from Fig. 1 are determined by (7).

$T r_{1} \times W+M_{0}=M_{1}$ 


$$
\begin{aligned}
M_{1}= & {\left[\begin{array}{llll}
1 & 0 & 0 & 0
\end{array}\right] \times\left[\begin{array}{cccc}
-1 & 0 & 0 & 1 \\
-1 & 0 & 0 & 1 \\
0 & -1 & -1 & 1 \\
1 & 1 & 1 & -1
\end{array}\right]+} \\
& +\left[\begin{array}{llll}
1 & 0 & 0 & 0
\end{array}\right]=\left[\begin{array}{llll}
0 & 0 & 0 & 1
\end{array}\right]
\end{aligned}
$$

B. Description of process of thermal power

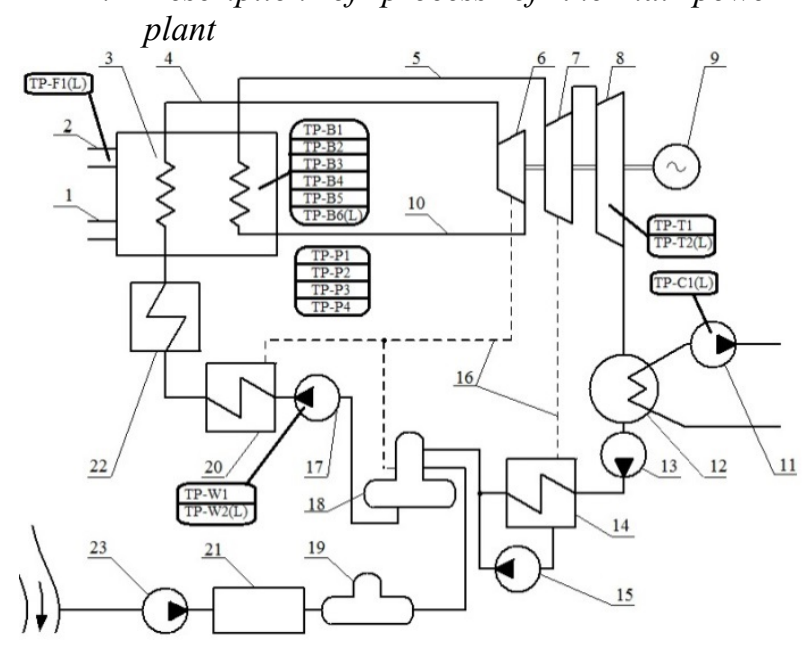

Fig. 2 A simplified diagram of an oil-gas fueled thermal power plant steam circuit

The same type equipment is shown once in the figure. Also there are not shown valves in the Fig. 2.

Fuel 1 and air 2 are supplied into steam generator (boiler) 3. Heated steam enters into turbine by steam pipes 4. Turbine at Fig. 2 consists of three cylinders (high pressure cylinder 6, medium pressure cylinder 7 and low pressure cylinder 8) that differ from each other by sizes and numbers of blades. There are also encountered turbines with one or two cylinders. Turbine rotates synchronous generator 9. Steam is partly taken for reheating by "cold" reheating pipe system 10 and then it is returned into turbine by "hot" reheating pipe system 5. Condensate comes into condenser 12 after turbine. Cooling water pumps 11 provide enough flow of cooling water from cooling tower or special pond (not shown at Fig. 2) to effectively take excess heat and convert steam into water. Group of condensate pumps 13 create head of water that is required to overcome the low-pressure heater 14 and get to deaerator 18. Deaerator removes dissolved gases out of water and gets water prepared to new cycle of heating. Moreover there is a system of pumps 23, chemical water treatment 22 and deaerator 19 that serves to compensate for the loss of water. Then feed water pumps 17 supplies feed water to the boiler through the medium-pressure heater 20 and high pressure heater 22. Also there are drain pump 15 and pipes of process steam extraction 16 .

Safety and effective work of such complex system as power plant steam circuit provides by
SCADA-systems that control station-service auxiliaries and regulate parameters of process. For supplying safety of equipment ant health of station staff emergency control schemes (also named technological protections) are responsible. Technological protections react to significant changes of parameters and carry out shutdown of equipment or load rejection. Parameters that are controlled by protections are:

- temperature, pressure, level of steam and water in boiler, turbine, condenser, deaerator;

- temperature, pressure, level of oil in lubrication system that deliver oil to bearings of electric motors and synchronous generator, turbine;

- vibration rate of turbine bearings;

- $\quad$ water and hydrogen consumption in cooling system of generator;

- operational condition of generator, pumps, induced fans, turbine;

- condition of control keys on emergency control panel.

There are list of groups of protections for Fig. 2.

- Protections of power unit:

o TP-P1 - protections that shutdown power unit;

o TP-P2 - protections that reject load of power unit to $50 \%$ of the nominal power;

o TP-P3 - protections that reject load of power unit to $30 \%$ of the nominal power or to idling;

o $\quad$ TP-P4 (L) P3 - local protections that regulate important parameters by opening and closing valves without changing behavior of power unit.

- Protections of boiler:

o TP-B1 - protections that shutdown boiler;

o $\quad$ TP-B2 - protections that reject load of boiler to $50 \%$ of the nominal power;

o $\quad$ TP-B3 - protections that reject load of boiler to $30 \%$ of the nominal power;

o TP-B4 - protections that shutdown fuel delivery to the boiler;

o TP-B5 (L) - local protections of boiler.

- Protections of steam turbine:

o TP-T1 - protections that shutdown turbine;

o TP-T2 (L) - local protections of turbine.

- Protections of feed water pumps: 
o TP-W1 - protections that shutdown feed water pumps; TP-W2 (L) local protections of feed water pumps.

- $\quad$ Protections of fuel delivery system:

o TP-F1 (L) - local protections.

- Protections of cooling water delivery system:

o TP-C1 (L) - local protections.

Structure and operating principle of the protections described in the operating instructions and other documents. But such description is not clear and does not allow to structure and study them from different positions. The next section presents a state model for power unit equipment and technological protections under the influence of changes in critical process parameters. The model is implemented in the form of Petri net.

\section{RESULTS AND DISCUSSION}

Information that was given above has been systematized into and processed in the Petri net (Fig. 3). The network presents several structural blocks. Left block represents the initiation of an emergency caused by a significant deviation from the parameters controlled by technological protections. Values of temperature, pressure and level of steam (water) can become less or more than allowable limits. It leads to changes in the right block.

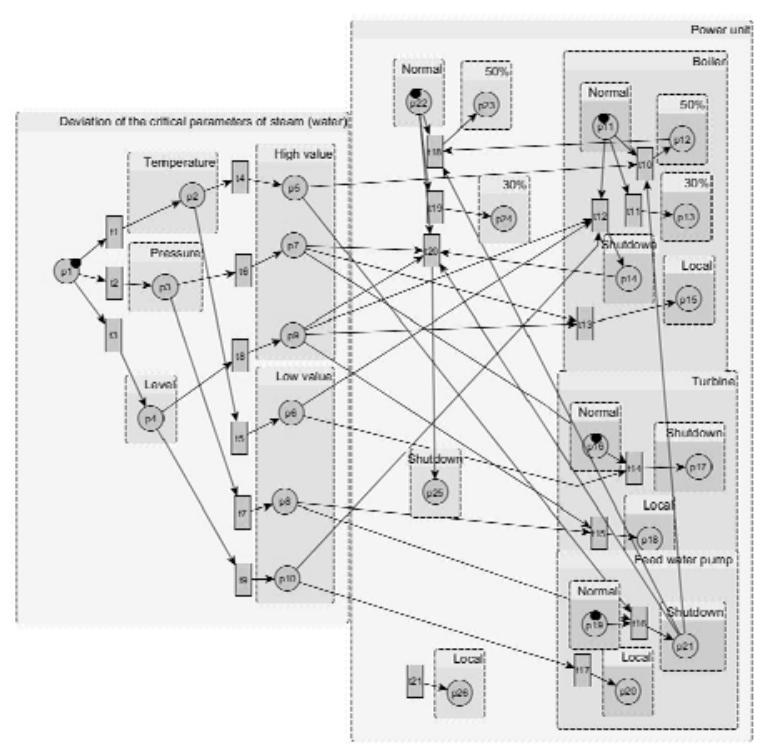

Fig. 3. Petri net of states of power unit equipment in an emergency change of parameters of steam (water).

Right block describes the equipment (boiler, turbine and feed water pump) set of states under the control of technological protections. Such states as normal state, loading by $50 \%$, loading by $30 \%$, safety shutdown the equipment and triggering of the local protections were identified for this model.

Normal states each component of equipment in initial marking have token. Another initial token is in place p1. Depending on what transition will fire deviation occurs with one of the three parameters.

The models for other critical characteristics (parameters of oil, vibration rate, operational condition of equipment and others) can be made in the same way. In addition to the visibility of the graphical representation of Petri nets analytical representation is interesting by enabling of creation software programs based on them. It was drawn incidence matrix with dimension $26 \times 21$ and initial marking for Petri net. Accident scenarios obtained in the form of sequence of firing transitions.

\section{CONCLUSIONS}

Energy companies applied great efforts for the creation of a modern safe and reliable electricity. But application of advanced technologies in the field of automation and informatization without the prior risk analysis does not always leads to effective safety improvement on the process. Using structures based on Petri nets allows to present sets of external events and internal states of the equipment as a dynamic multilevel model.

Currently, authors are developing a software package for the analysis of the risk of accidents at thermal power plants on the basis of these models.

\section{ACKNOWLEDGMENTS}

This study is supported by the Foundation for Assistance to Small Innovative Enterprises in the scientific and technical field (FASIE).

\section{REFERENCES}

[1] V. Belov, B. Pergamenschik, "Major accidents involving thermal power plant and their influence on the layout solutions the main buildings," Vestnik MGSU [Bulletin of MGSU], vol. 4, pp. 61-69, 2013. (in Russian)

[2] V. Egorov and S. Trashchenkov, Risk analysis of an accident on the process of thermal power plants Methodological problems in reliability study of large energy systems. Session 86. Reliability of Liberalized Energy Systems, June 31 - July 4, 2014, St-Petersburg, Russia. Irkutsk: ESI SB RAS, 2015. (in Russian)

[3] M.M. Mansour, Mohamed A.A. Wahab, and Wael M. Soliman, "Petri nets for fault diagnosis of large power generation station,” Ain Shams Engineering Journal, vol. 4(4), pp. 831 - 842, 2013.

[4] Tavana, M. "Dynamic process modelling using Petri nets with applications to nuclear power plant emergency management", Int. J. Simulation and Process Modelling, Vol. 4, No. 2, pp.130-138, 2008.

[5] L. Wehenkel. "Emergency Control and Its Strategies", Proc of PSCC 1999, Vol. 1, pp. 35-48. Trondheim, Norway, June 28July 2, 1999.

[6] Peterson, J.L. Petri Net Theory and the Modelling of Systems, Prentice-Hall, Inc., Morristown, NJ, 1981.

[7] Zurawski R, Zhou M. Petri nets and industrial application: a tutorial. IEEE Trans Indus Electron, Vol. 41(6), pp. 567-583, 1994.

[8] Sum J, Qin S-Y, Song Y-H. Fault diagnosis of electric power systems based on fuzzy Petri nets. IEEE Trans Power System, Vol. 19, pp. 2053-2059, 2004. 
[9] Wang J. Petri nets for dynamic event-driven system modeling. In: Fishwick Paul A, editor. Handbook of dynamic system modeling. Chapman\& Hall/CRC; 2007.

[10] Murata, T. Petri nets: Properties, analysis and applications. Proceedings of the IEEE, Vol. 77(4), pp. 541-580, 1989.

[11] Viswanadham, N. and Srinivasa Raghavan, N.R. 'Performance analysis and design of supply chains: a petri net approach', Journal of the Operational Research Society, Vol. 51, No. 10, pp.1158-1169, 2000.

[12] Zhou, M. C., and F. DiCesare Adaptive design of Petri net controllers for error recovery in automated manufacturing systems. IEEE Trans. on Systems, Man, and Cybernetics Vol. 19(5), pp. 963-973, 1989.

[13] Ajmone Marsan, M. Stochastic Petri nets: an elementary introduction. Advances in Petri Nets, LNCS, p. 424, 1990.

[14] Desrochers, A., and R. Ai-Jaar. Applications of Petri Nets in Manufacturing Systems: Modeling, Control and Performance Analysis. IEEE Press, 1995.
[15] Genrich, J.H., and K. Lautenbach, System modeling with high-level Petri nets. Theoretical Computer Science Vol. 13, pp. 109-136, 1981.

[16] Jensen, K., Coloured Petri Nets. Basic Concepts, Analysis Methods and Practical Use (3 volumes). London: SpringerVerlag, 1997.

[17] Ramamoorthy, C., and G. Ho. Performance evaluation of asynchronous concurrent systems using Petri nets. IEEE Transaction on Software Engineering, Vol. 6(5), pp. 440-449, 1980.

[18] Desel J, Esparza J. Free choice petri nets. UK: Cambridge University Press; p. 256, 1995.

[19] Jingbo H, Longhua M. Fault diagnosis of substation based on Petri nets technology. International conference on power system technology, October 2006. p. 1-5.

[20] Peng MW, Liu Y, Guo CX, Huang B, Zhao W. A new fault diagnosis approach in power system based on elementoriented Petri nets with time-stamp. International conference on sustainable power generation and supply, SUPERGEN'09, Nanjing, April 2009, p. 1-7. 